\title{
Myasthenia Class V
}

National Cancer Institute

\section{Source}

National Cancer Institute. Myasthenia Class V. NCI Thesaurus. Code C112018.

Severe muscle weakness that requires intubation, with or without mechanical ventilation. 